\title{
Identification of inhibitors of SARS-CoV-2 in-vitro cellular toxicity in human (Caco-2) cells using a large scale drug repurposing collection
}

\section{Bernhard Ellinger ( $\nabla$ corona-repurposing@ime.fraunhofer.de )}

Fraunhofer Institute for Molecular Biology and Applied Ecology IME, Branch for Translational Medicine \& Pharmacology, ScreeningPort, Hamburg, Germany

\section{Denisa Bojkova}

University Hospital Frankfurt, Frankfurt am Main, Germany

\section{Andrea Zaliani}

Fraunhofer Institute for Molecular Biology and Applied Ecology IME, Branch for Translational Medicine \& Pharmacology, ScreeningPort, Hamburg, Germany

Jindrich Cinatl

University Hospital Frankfurt, Frankfurt am Main, Germany

\section{Carsten Claussen}

Fraunhofer Institute for Molecular Biology and Applied Ecology IME, Branch for Translational Medicine \& Pharmacology, ScreeningPort, Hamburg, Germany

\section{Sandra Westhaus}

University Hospital Frankfurt, Frankfurt am Main, Germany

\section{Jeanette Reinshagen}

Fraunhofer Institute for Molecular Biology and Applied Ecology IME, Branch for Translational Medicine \& Pharmacology, ScreeningPort, Hamburg, Germany

\section{Maria Kuzikov}

Fraunhofer Institute for Molecular Biology and Applied Ecology IME, Branch for Translational Medicine \& Pharmacology, ScreeningPort, Hamburg, Germany

\section{Markus Wolf}

Fraunhofer Institute for Molecular Biology and Applied Ecology IME, Branch for Translational Medicine \& Pharmacology, ScreeningPort, Hamburg, Germany

\section{Gerd Geisslinger}

Pharmazentrum Frankfurt/ZAFES, Institut für Klinische Pharmakologie, Klinikum der Goethe- Universität Frankfurt, Frankfurt am Main, Germany

\section{Philip Gribbon}

Fraunhofer Institute for Molecular Biology and Applied Ecology IME, Branch for Translational Medicine \& Pharmacology, ScreeningPort, Hamburg, Germany

\section{Sandra Ciesek}




\section{Research Article}

Keywords: SARS-CoV-2, compound screening, inhibition of viral induced cytotoxicity, human epithelial colorectal adenocarcinoma cell line Caco-2

Posted Date: April 20th, 2020

DOI: https://doi.org/10.21203/rs.3.rs-23951/v1

License: (c) (i) This work is licensed under a Creative Commons Attribution 4.0 International License. Read Full License 


\section{Abstract}

To identify possible candidates for progression towards clinical studies against SARS-CoV-2, we screened a well-defined collection of 5632 compounds including 3488 compounds which have undergone clinical investigations (marketed drugs, phases $1-3$, and withdrawn) across 600 indications. Compounds were screened for their inhibition of viral induced cytotoxicity using the human epithelial colorectal adenocarcinoma cell line Caco-2 and a SARS-CoV-2 isolate. The primary screen of 5632 compounds gave 271 hits. A total of 64 compounds with IC50 $<20 \mu \mathrm{M}$ were identified, including 19 compounds with IC50< $1 \mu \mathrm{M}$. Of this confirmed hit population, $90 \%$ have not yet been previously reported as active against SARS-CoV-2 in-vitro cell assays. Some 37 of the actives are launched drugs, 19 are in phases 1-3 and 10 pre-clinical. Several inhibitors were associated with modulation of host pathways including kinase signaling P53 activation, ubiquitin pathways and PDE activity modulation, with long chain acyl transferases were effective viral inhibitors.

\section{Introduction}

The Coronaviridae are a family of positive single stranded encapsulated viruses. They typically cause mild respiratory diseases, but infections with the ß-coronavirus SARS-CoV, MERS and SARS-CoV-2 can lead to acute respiratory diseases and high mortality, particularly in individuals with underlying health conditions. In the last 20 years, Coronaviridae have emerged in two severe outbreaks, 2002/2003 with SARS-CoV, 2012 with MERS and one pandemic, in late 2019 with SARS-CoV-2. At the time of writing, the coronavirus SARS-Cov-2 pandemic has led to close to 1 million confirmed infections and tens of thousands deaths (https://coronavirus.jhu.edu). Multiple interventional clinical trials have been initiated in the search for effective pharmacological treatments against SARS-CoV-2 infection and the related disease Covid-19(7). The selection of single drug or combination treatments for these studies have been based on previously described activities against SARS-CoV, Ebola, HIV and Malaria. Thus, drugs under investigation include remdesivir, interferons, ribavirin, lopinavir/ritonavir and chloroquine(s)(2). Bioinformatics analyses have also proposed additional repurposed drugs based on the interactome between viral encoded proteins and host-cell pathways(3). In the absence of safe and effective vaccines against SARS-CoV-2, repurposing of existing drugs represents a first pragmatic strategy for the treatment of Covid-19 patients. Regardless of the origin of the therapeutic, however, properly controlled clinical safety and efficacy studies are still essential pre-requisites before approval of pharmacological treatments for Covid-19, even with repurposed drugs.

To identify possible candidates for progression towards clinical studies against SARS-CoV-2, we screened a well-defined collection of 5632 compounds including 3488 compounds which have undergone clinical investigations (marketed drugs, phases $1-3$, and withdrawn) across 600 indications(4). The set was established as a mirror of that reported by the Broad Institute(4). Compounds were screened for their inhibition of viral induced cytotoxicity using the human epithelial colorectal adenocarcinoma cell line Caco-2 and a SARS-CoV-2 isolate obtained from an individual originally exposed to the virus in the Wuhan region of China(5). The primary assay is based on a recent manuscript(6) and has been further 
optimised to a high content cell imaging format, compatible with medium throughput screening (Figure S1). Primary screening was performed at $10 \mu \mathrm{M}$ compound concentration, at a virus multiplicity of infection (MOI) of 0.01 and a virus incubation period of $48 \mathrm{~h}$, to ensure multiple viral replication cycles. High content screening assay readouts of cell viability used digital phase imaging (label free) and fluorescent quantification of stained nuclei. Selected hits were then confirmed using 8-point dose concentration response in anti-viral and cytotoxicity assays.

\section{Results}

Pharmacological validation used four compounds (cycloheximide, emetine, remdesivir and chloroquine), previously reported to inhibit either SARS-COV-2, SARS-COV or MERS virus cytotoxicity in phenotypic readouts using the Vero- 6 kidney epithelial cell line, originally isolated from an African green monkey, (Figure S2 and references therein). The IC50 values for emetine $(0.52 \pm 0.09 \mu \mathrm{M})$ and cycloheximide $(0.58$ $\pm 0.02 \mu \mathrm{M}$ ) were elevated compared to reported activities of these compounds against MERS and SARS (range 0.04 to $0.18 \mu \mathrm{M})$. The IC50 of the RNA polymerase inhibitor, remdesivir, $(0.76+/-0.18 \mu \mathrm{M})$ was similar to that in recent reports $(0.77)(7)$. Chloroquine was not active in the Caco-2 cell assay (IC50> 20 $\mu \mathrm{M})$, a result which differs from several reports of its activity against Vero- 6 cells in the micromolar range (Figure S2). This may be due to differences in the use of human versus monkey cell line, or in the respective viral strains and was also reflected in results from the primary screen, where chloroquine and related compounds were mostly inactive (Table S2).

The primary screen of 5632 compounds gave 271 hits (inhibition cut-off 75\%). A group of 184 compounds were selected based on clinical status and mechanism of action and then profiled in concentration response (Figure 1A). The hepatitis $C$ anti-viral, alisporivir, (not in the screened set), was added at this stage. Primary screen quality was acceptable with an average $Z^{\prime}$ value of 0.75 (Figure 1B). The calculated inhibition values for virus induced cytotoxicity correlated well $\left(R^{2}=0.88\right)$ between digital phase and nuclear staining readouts (Figure 1C). Inhibition calculated from digital phase parameters was used for further quantification of compound antiviral activities. Analysis of the annotated targets of the primary hit population of 271 compounds indicates an enrichment of kinase inhibitors and relative reduction in GPCR modulating compounds (Figure 1D). Consistent with this result, the relative cellular localisation of the primary targets of the hit compounds is elevated for the intracellular compartment (Figure S2). Plates in dose response studies were controlled by $Z^{\prime}$ determination and had Z' $>0.4$ in all cases.

A total of 64 compounds with IC $50<20 \mu \mathrm{M}$ were identified in the primary screen, including 19 compounds with IC50 $<1 \mu \mathrm{M}$ (Table 1 and Figure S4). Of this confirmed hit population, 90\% have not yet been previously reported as active against SARS-CoV-2 in-vitro cell assays. Some 35 of the actives are launched drugs, 19 are in phases 1-3 and 10 pre-clinical.

From the library screen hits (IC50 $<20 \mu \mathrm{M}$ ) we flagged 6 compounds (camostat, nafamostat, lopinavir, mefloquine, papaverine, cetylpyridinium) which had previously been reported to show activity in either 
SARS-Cov-2, SARS-Cov or MERS phenotypic assays using Vero-6, Calu-3 (human lung) or BHK-21 (hamster kidney fibroblast) cells (Figure S3). The reported inhibitors of the viral entry protease TMPRSS-2 (camostat and nafamostat) appear some 100x more potent against SARS-Cov-2 in Caco-2 cells, compared to Vero-6 cells. However, relative to MERS and SARS-CoV, (in Calu-3 cells), the IC50 values of the compounds are comparable. The IC50 values of lopinavir and mefloquine are relatively consistent across assays, with less than 4-fold shifts between all cell and virus types. Papaverine is 8-fold more potent against SARS-Cov-2 (in Caco-2) than against MERS (in BHK-21), whilst cetylpyridinium activity is similar in both assays. From this result, the Caco- 2 based screening assay appears to be pharmacologically relevant and suitable for identifying compounds with diverse mechanisms of action. Results with Caco-2 seem more closely aligned to previous data seen for human cell lines (Calu-3), compared to non-human cells (Vero-6 or BHK-21), and in particular for inhibitors associated with virus entry.

Dose dependent viral inhibition was not observed with the reference compound, chloroquine (Figure S2), although the related antimalarial, mefloquine, was identified in the primary screen and was moderately active $(\mathrm{IC} 50=14.1 \mu \mathrm{M})$ in concentration response. The anti-malarial tafenoquine and pyronaridine were inactive in dose response (Figure S3) and, along with hydroxychloroquine, they were inactive in the primary screen (Table S1). This discrepancy may be linked to a greater sensitivity of Vero- 6 cells to this class of compound, or associated with the limited solubility of this class of drugs.

A recently published experimental and bioinformatics-informed analysis suggested a set of 66 druggable human proteins or host factors associated with SARS-CoV-2a and highlighted 69 compounds in clinical or pre-clinical development, which were predicted to have activity(3). From this group 52 compounds or close analogues (Tanimoto >0.9) were screened in this study. Of the 52 compounds, 7 were classified as active in the primary assay (Table S2) a success rate of $14 \%$. From the 7 hits, 5 were selected for concentration response and 4 compounds were active (IC50 < $20 \mu \mathrm{M})$, (Table S2, Figure S4).

\section{Discussion}

The activity of the reference compound, remdesivir $(I C 50=0.76 \mu \mathrm{M})$ was confirmed in this study. Remdesivir targets the viral nsp12 RNA-dependent RNA polymerase ${ }^{(8)}$ and is currently under evaluation in an adaptive, randomized, double-blind, placebo-controlled phase III clinical trial ${ }^{(9)}$. Nafamostat (IC50 = $0.04 \mu \mathrm{M})$ is thought to target processing of virus spike proteins, inhibiting membrane fusion(9).

Tioguanine $(\mathrm{IC50}=1.71 \mu \mathrm{M})$ has been reported as an inhibitor of the viral protease PL-Pro in MERS and SARS-CoV ${ }^{(10)}$. One possibility is that tioguanine is phosphorylated leading to a failure of faithful RNA copying. Camostat was also reported to inhibit the entry of SARS-CoV-2 in CaCo-2 cells by targeting the serine protease TMPRSS2(11). These results indicate that the assay procedure is able to confirm the activity of clinically relevant compounds suggested to be active against both viral entry and replication mechanisms. Of the HIV aspartyl protease inhibitors currently being evaluated in clinical trials for SARSCoV-2, lopinavir was active only at the highest concentration in the dose response (Figure S4), and 
ritonavir and darunavir were inactive at the primary screening stage (Table S1), suggesting this class of enzyme may not play a role in viral processing. Several of the more active compounds (Table 1) showed a relatively small difference between the cytotoxic and antiviral IC50 values with $\mathrm{Cl}$ values $<3$ (eg., thioguanosine, amuvatinib, cetylpyridinium and sorafenib). Many of these compounds have detailed data available on their pharmacokinetics and safety in human subjects and patients. It will be important to use this information to establish whether a sufficiently high concentration can be safely achieved at the site of action, presumably the lung epithelium, before proposing follow on clinical studies.

The antimalarials chloroquine and its close relative hydroxychloroquine are reported to show antiviral activity against SARS-CoV(9). These are now being tested either as monotherapy or combination therapy in clinical trials (clinicaltrials.gov updated 30.03.2020). With the Caco-2 cell line, chloroquine diphosphate was inactive as a reference (Figure S2). The analogue mefloquine, $($ IC50 $=14.1 \mu \mathrm{M})$, showed inhibition in dose response, while tafenoquine and amodiaquine were inactive (Figure S4). Similarly, lumefantrine and primaquine, both commonly used anti-malarials, were inactive (Table S1). This discrepancy with chloroquine may be due to the differences in the origin of the cell lines used in the different in-vitro measurements. Additional in-vitro studies using relevant human cell lines or, ideally, primary tissue explant models are needed to clarify chloroquine's anti SARS-Cov-2 properties in-vitro.

Several antifungal compounds were identified, with chlormidazole, ravuconazole, posaconazole and ketoconazole having IC50's in the range of $2 \mu \mathrm{M}$. In addition, cloconazole, and oxiconazole gave IC50 values at $20 \mu \mathrm{M}$ or less (Table 1 and Figure S4), although results showed some cytotoxicity in the same range. Reports of antiviral activity for this class of compounds are uncommon, but exceptions include posaconazole against parechovirus A3(12). The effective concentrations are an order of magnitude higher than those reported for the antifungal activity in-vitro. Similar membrane interacting molecules have been reported to interfere with virus-host membrane fusion mechanisms(13) and this may be a potential mechanism of action for this compound class.

Dapivirine $(\mathrm{IC50}=0.73 \mu \mathrm{M})$ is a non-nucleoside reverse transcriptase inhibitor developed as an anti-HIV agent. It has been recently shown to have broad antiviral activity with in vitro micromolar IC50 values against influenza $A$ and $B(14)$. Almitrine (IC50 $=1.42 \mu \mathrm{M})$ was authorized for COPD later withdrawn after risk benefit balance reassessment(15). This compound was selected, among others, after a virtual screening analysis against the SARS-CoV-2 viral protease Mpro(16) and, following additional confirmatory in-vitro and in-vivo studies, may be a viable candidate for further development. The orally available farnesyl transferase inhibitor ionafarnib $($ IC50 $=5.68 \mu \mathrm{M})$ has been studied in renal carcinoma(17) and has anti-viral activity in hepatitis $D(18)$.

The crystallization of SARS-CoV-2 spike protein complexed to Angiotensin-Converting Enzyme-2 (ACE2) [PDB code $=6 \mathrm{M} 18](19)$, has shed new light on possible SARS-CoV-2 virus entry mechanisms modulating virus-host interactions. ACE2 is expressed in Caco-2, Calu-3 and Vero- 6 cells on the apical membrane domains (20). A recent report pointed to the potential for elevated ACE-2 expression levels caused by modulation of the ACE/ACE2/AT1R/TMPRSS2 system by "sartans" class compounds (e.g., candesartan, 
telmisartan,) or ACE inhibitors (e.g. captopril, or the pro-drug enalapril). Such inhibitors might, paradoxically, be either beneficial or deleterious for Covid-19 patients(21). AT1R inhibitors may induce ACE2 beneficially but at the expense of facilitating viral entry. In our study, we were unable to identify either classical ACE inhibitors such as Captopril or classical AT1R inhibitor "sartans" as inhibitors in the primary screen (Table S1).

Comparison of the recently proposed interactome compounds(3), with the set screened in this study showed 40 identical and 12 similar compounds (Table S2). The 5 profiled compounds from the 7 hits were: loratadine $(\mathrm{IC} 50=15.13 \mu \mathrm{M})$; nafamostat $($ IC50 $=0.04 \mu \mathrm{M})$, pevonedistat $($ IC50 $=0.63 \mu \mathrm{M})$; camostat $($ IC50 $=0.64 \mu \mathrm{M})$; and idarubicin $($ IC50 $>20 \mu \mathrm{M})$. The corresponding identified biochemical targets were: Serine proteases (nafamostat and camostat); Histamine 1 receptor (loratadine); Topoisomerases (idarubicin/daunorubicin); and the NEDD-8 activating enzyme (pevonedistat). The lack of activity of the predicted compound ligands, for the majority of the originally identified pathways, may be due to target expression levels, compound solubility or chemical/metabolic stabilities.

The relatively potent pre-clinical compound, NSC319276 (IC50 $<0.02 \mu \mathrm{M})$ has been reported to elevate intracellular $\mathrm{Zn}^{2+}$, leading to modulation of $\mathrm{p} 53$ folding(22). The compound also interferes with transition metal metabolism, inducing oxidative stress and depletion of nucleotide reserves, suggesting a possible mechanism for inhibiting viral replication. LY2228820, (IC50 $=0.87 \mu \mathrm{M})$ also known as ralimetinib, is a potent and selective inhibitor of $a$ and $\beta$ isoforms of p38 MAPK in vitro(23). Ralimetinib, was involved in a stopped phase 2 clinical trial for tamoxifen-resistant ovarian cancer(24). Papaverine (IC50 $=1.1 . \mu \mathrm{M})$ is a non-narcotic alkaloid for heart disease, impotency, and psychosis. It was recently found to be an effective inhibitor of multiple strains of influenza virus. Papaverine is a phosphodiesterase (PDE) inhibitor(25), like ethaverine $($ IC50 $=0.64 \mu \mathrm{M})$ and drotaverine $($ IC50 $=6.07 \mu \mathrm{M})$ which both inhibit PDE-4 $(26)$.

Hematoporphyrin (IC50 $=1.85 \mu \mathrm{M})$ is a photodynamic activated DNA intercalator and single strand breaker with anticancer activity(27), which also shows antiviral effects via DNA polymerase inactivation(28). SB- $612111(\mathrm{IC} 50=0.77 \mu \mathrm{M})$ has been developed as a potent and selective NOP antagonist(29) and binds the Opiate and Adenosine receptor proteins(29). Amuvatinib was one of the most potent compounds identified (IC50 $=0.02 \mu \mathrm{M})$ but was unsuccessful in trials for solid tumors and small cell lung carcinoma, but was well tolerated(30). Amuvatinib is a multi-targeted tyrosine kinase inhibitor (c-MET, c-RET and the mutant forms of c-KIT, PDGFR and FLT3). Additional tyrosine kinase inhibitors identified were sorafenib (IC50 $=1.55 \mu \mathrm{M})$, regorafenib $($ IC50 $=1.67 \mu \mathrm{M})$, pexidartinib $(5.43)$ and vatalanib $(I C 50=18.27 \mu \mathrm{M})$ suggesting a role for kinase signaling in the receptor mediated host response to SARS-Cov-2.

\section{Conclusions}

We have tested an extended library of repurposed compounds and profiled reference compounds, using the Caco-2 epithelial cell line and a live virus SARS-Cov-2 clinical isolate. This analysis has confirmed the in-vitro activities, in a human cell line, for multiple compounds previously identified as potential Covid-19 
therapeutics (remdesivir, camostat, nafamostat, lopinavir, mefloquine, thioguanosine and tioguanine). These data indicate that preventing viral infection by modulating viral spike protein related mechanisms and/or protease activities and fusion, may play a key role in preventing viral entry and replication.

The most important new finding is the identification of a tranche of over 50 compounds with potential alternative mechanisms of action against SARS-CoV-2 in-vitro. This group includes several inhibitors associated with modulation of host pathways including kinase signaling (e.g. amuvatinib, sorafenib, regorafenib and pexidartinib), P53 activation (NSC319726), ubiquitin pathways (VLX600) and PDE activity modulation (papaverine, drotaverine and ethaverine). The long chain acyl transferases have been implicated(3) in virus entry and in our hands lonafarnib and "azole" family compounds, were effective viral inhibitors, although at concentrations an order of magnitude higher than typical antifungal activities

The compounds used in this study are well annotated with respect to primary and secondary targets and we hope that these meta-data will be used with our results and the findings of other labs to help identify additional treatment opportunities, including drug combinations. It will be key to determine whether any of these clinical-stage compounds or related molecules could safely achieve active concentrations at the targeted site, human lung epithelia. With a further combination of in-vitro activity and tissue distribution, it may be possible to make suggestions about which would be fruitful avenues for future Covid-19 preclinical and clinical studies.

\section{Declarations}

Acknowledgments

We thank Timothy Wells of the Medicine for Malaria Venture for critical reading of the manuscript and providing valuable insight into repurposing approaches. We acknowledge funding from the Johanna Quandt Universitäts-Stiftung. This work has been supported, in part, by the LOEWE Center for Translational Medicine and Pharmacology (TMP) and the Fraunhofer cluster of excellence for immune mediated diseases. FAIRification of data for community use was supported by funding from the European Union's Horizon 2020 programme of EOSC-LIFE, under grant agreement number 824087. We thank Andrew Leach, Anna Gaulton, Anne Hersey, Eloy Felix, Emma Manners and Patricio Bento of the European Bioinformatics Institute (Hinxton) for preparing FAIR data sets. We thanks Yulia Gerhardt and

Peter Maas of SPECS and Joshua Bitker (ex-Broad) for input into the selection and quality control of the compound library. We thank Giuseppe Vangelista for infrastructural support.

\section{Contributions}

SC, JC, CC, GG, BE, AZ and PG conceived the study. DB, SW, MK, BE, JC, MK and MW carried out tissue culture work, virus experiments, and cytotoxicity assays. AZ, BE, MK and JR performed data analysis. SC 
and CC, supervised the work. PG, AZ, DB and BE prepared the initial manuscript, with contributions received from all authors. All authors read and approved the final manuscript.

Competing interests

The authors declare no competing interests.

Materials \& correspondence

Correspondence and material requests to Bernhard Ellinger and Sandra Ciesek. coronarepurposing@ime.fraunhofer.de

\section{References}

1. Kupferschmidt, J. Cohen, Race to find COVID-19 treatments accelerates. Science (New York, N. Y.). 367, 1412-1413 (2020), doi:10.1126/science.367.6485.1412.

2. Li, E. de Clercq, Therapeutic options for the 2019 novel coronavirus (2019-nCoV). Nature reviews. Drug discovery. 19, 149-150 (2020), doi:10.1038/d41573-020-00016-0.

3. E. Gordon et al., A SARS-CoV-2-Human Protein-Protein Interaction Map Reveals Drug Targets and Potential Drug-Repurposing (2020).

4. M. Corsello et al., The Drug Repurposing Hub: a next-generation drug library and information resource. Nature medicine. 23, 405-408 (2017), doi:10.1038/nm.4306.

5. Hoehl et al., Evidence of SARS-CoV-2 Infection in Returning Travelers from Wuhan, China. The New England journal of medicine. 382, 1278-1280 (2020), doi:10.1056/NEJMc2001899.

6. Bojkova et al., SARS-CoV-2 infected host cell proteomics reveal potential therapy targets (2020).

7. P. Sheahan et al., Broad-spectrum antiviral GS-5734 inhibits both epidemic and zoonotic coronaviruses. Science translational medicine. 9 (2017), doi:10.1126/scitranslmed.aal3653.

8. L. Agostini et al., Coronavirus Susceptibility to the Antiviral Remdesivir (GS-5734) Is Mediated by the Viral Polymerase and the Proofreading Exoribonuclease. mBio. 9 (2018), doi:10.1128/mBio.0022118.

9. Wang et al., Remdesivir and chloroquine effectively inhibit the recently emerged novel coronavirus (2019-nCoV) in vitro. Cell research. 30, 269-271 (2020), doi:10.1038/s41422-020-0282- 0.

10. -W. Cheng et al., Thiopurine analogs and mycophenolic acid synergistically inhibit the papain-like protease of Middle East respiratory syndrome coronavirus. Antiviral research. 115, 9-16 (2015), doi:10.1016/j.antiviral.2014.12.011. 
11. Hoffmann et al., SARS-CoV-2 Cell Entry Depends on ACE2 and TMPRSS2 and Is Blocked by a Clinically Proven Protease Inhibitor. Cell (2020), doi:10.1016/j.cell.2020.02.052.

12. Rhoden, W. A. Nix, W. C. Weldon, R. Selvarangan, Antifungal azoles itraconazole and posaconazole exhibit potent in vitro antiviral activity against clinical isolates of parechovirus A3 (Picornaviridae). Antiviral research. 149, 75-77 (2018), doi:10.1016/j.antiviral.2017.11.011.

13. Speerstra et al., Antivirals acting on viral envelopes via biophysical mechanisms of action. Antiviral research. 149, 164-173 (2018), doi:10.1016/j.antiviral.2017.11.018.

14. Hu et al., Discovery of dapivirine, a nonnucleoside HIV-1 reverse transcriptase inhibitor, as a broadspectrum antiviral against both influenza A and B viruses. Antiviral research. 145, 103-113 (2017), doi:10.1016/j.antiviral.2017.07.016.

15. EMA - European Medicines Agency, "Assessment report for almitrine-containing medicinal products for oral use, EMA/332160/2013" EMA/332160/2013. (EMA, 23 May 2013; https://www.ema.europa.eu/en/documents/referral/almitrine-article-31-referral-prac- assessmentreport_en.pdf).

16. Wu et al., Analysis of therapeutic targets for SARS-CoV-2 and discovery of potential drugs by computational methods. Acta Pharmaceutica Sinica B (2020), doi:10.1016/j.apsb.2020.02.008.

17. Wang et al., Synergistic effect of farnesyl transferase inhibitor lonafarnib combined with chemotherapeutic agents against the growth of hepatocellular carcinoma cells. Oncotarget. 8, 105047-105060 (2017), doi:10.18632/oncotarget.22086.

18. Rizzetto, A. Ciancio, The prenylation inhibitor, lonafarnib: a new therapeutic strategy against hepatitis delta. The Lancet Infectious Diseases. 15, 1119-1120 (2015), doi:10.1016/S1473- 3099(15)00155-3.

19. Yan et al., Structural basis for the recognition of SARS-CoV-2 by full-length human ACE2. Science (New York, N.Y.). 367, 1444-1448 (2020), doi:10.1126/science.abb2762.

20. Ren et al., Analysis of ACE2 in polarized epithelial cells: surface expression and function as receptor for severe acute respiratory syndrome-associated coronavirus. The Journal of general virology. 87, 1691-1695 (2006), doi:10.1099/vir.0.81749-0.

21. Gurwitz, Angiotensin receptor blockers as tentative SARS-CoV-2 therapeutics. Drug development research (2020), doi:10.1002/ddr.21656.

22. Nguyen, W. Liao, S. X. Zeng, H. Lu, Reviving the guardian of the genome: Small molecule activators of p53. Pharmacology \& therapeutics. 178, 92-108 (2017), doi:10.1016/j.pharmthera.2017.03.013.

23. M. Campbell et al., Characterization of LY2228820 dimesylate, a potent and selective inhibitor of p38 MAPK with antitumor activity. Molecular cancer therapeutics. 13, 364-374 (2014), doi:10.1158/1535-7163.MCT-13-0513.

24. Patnaik et al., A First-in-Human Phase I Study of the Oral p38 MAPK Inhibitor, Ralimetinib (LY2228820 Dimesylate), in Patients with Advanced Cancer. Clinical cancer research : an official journal of the American Association for Cancer Research. 22, 1095-1102 (2016), doi:10.1158/10780432.CCR-15-1718. 
25. Aggarwal, G. P. Leser, R. A. Lamb, Repurposing Papaverine as an Antiviral Agent against Influenza Viruses and Paramyxoviruses. Journal of virology. 94 (2020), doi:10.1128/JVI.01888-19.

26. V. Muravyov et al., Hemorheological efficiency of drugs, targeting on intracellular phosphodiesterase activity: In vitro study. Clinical Hemorheology and Microcirculation. 36, 327-334 (2007).

27. P. Boegheim, T. M. Dubbelman, L. H. Mullenders, J. van Steveninck, Photodynamic effects of haematoporphyrin derivative on DNA repair in murine L929 fibroblasts. The Biochemical journal. 244, 711-715 (1987), doi:10.1042/bj2440711.

28. Munson BR, Fiel RJ, Hematoporphyrin-sensitized photodynamic inactivation of viral RNA-dependent DNA polymerase. Res Commun Chem Pathol Pharmacol. 16, 175-178 (1977).

29. K. Tosh et al., Repurposing of a Nucleoside Scaffold from Adenosine Receptor Agonists to Opioid Receptor Antagonists. ACS omega. 3, 12658-12678 (2018), doi:10.1021/acsomega.8b01237.

30. A. Byers et al., A phase 2, open-label, multi-center study of amuvatinib in combination with platinum etoposide chemotherapy in platinum-refractory small cell lung cancer patients. Oncotarget. 8, 81441-81454 (2017), doi:10.18632/oncotarget.19888.

\section{Methods}

The collection of 5641 compounds was assembled by an external partner (SPECS) in a manner aligned to the recommendations from the Broad Institute (Cambridge Mass., USA) ${ }^{1}$. In assembling this "mirror" collection, compounds were purchased from the same set of $>70$ high quality suppliers identified by the Broad, (personal communication Dr Joshua Bitker), and quality controlled by LC/MS for purity and identity (minimum purity $>90 \%$ ). Compounds at $10 \mathrm{mM}$ were stored in $100 \% \mathrm{DMSO}$ at $-20^{\circ} \mathrm{C}$. A curated database is available containing the compound, indication, primary target (where known) and mechanism of action, as well as analysis tools which can assist in mechanism of action determination and target elucidation ${ }^{1}$. These data have also been made available through ChEMBL resource.

\section{Cell culture}

Human epithelial cell line, derived from colon carcinoma, Caco-2 cells, were obtained from the Deutsche Sammlung von Mikroorganismen und Zellkulturen (DSMZ). Cells were grown in Minimal Essential Medium (MEM) supplemented with $10 \%$ fetal bovine serum (FBS) and containing $100 \mathrm{lU} / \mathrm{ml}$ penicillin and $100 \mu \mathrm{g} / \mathrm{ml}$ streptomycin at $37^{\circ} \mathrm{C}$. All culture reagents were purchased from Sigma.

\section{Virus culture}


SARS-CoV-2 was isolated from samples of travelers returning from Wuhan (China) to Frankfurt (Germany) using Caco-2 cells as described previously ${ }^{2}$. SARS-CoV-2 stocks used in the experiments had undergone one passage on Caco- 2 cells and were stored at $-80^{\circ} \mathrm{C}$. Virus titers were determined as $\mathrm{TCID} 50 / \mathrm{ml}$ in confluent cells in 96-well microtiter plates.

\section{Cell viability assays for primary screening and dose response studies}

To determine compound antiviral activity, we adapted a previously published protocol ${ }^{2}$. The screening assay workflow is shown in Figure S1. Compounds were added to confluent layers of Caco- 2 cells in MEM supplemented with $1 \%$ FBS in 96 -well plates. For the primary screen final compound concentration was $10 \mu \mathrm{M}(0.1 \% \mathrm{DMSO}$ final) in singlicate. Dose response profiling of selected priority compounds was performed with a range of eight different concentrations in three independent replicates (maximum 20 $\mu \mathrm{M}$, minimum $20 \mathrm{nM}$, half log dilution factor, $0.1 \%$ DMSO final). Following the addition of compounds, cells were immediately infected with SARS-CoV-2 at MOI 0.01 . Control wells (+ virus and - virus) also contained DMSO at $0.1 \%$ DMSO final. After 48 hours, cells were fixed using 3 \% PFA in PBS, and the plates sealed and disinfected to inactivate SARS-CoV-2 ${ }^{3}$. Quantification of viral inhibition (based upon Caco-2 cell viability relative to controls) was performed using high content imaging (PerkinElmer, Operetta CLS). Firstly, in label free mode using digital phase contrast, with maximum contrast as a read out ${ }^{4}$. Secondly, cell nuclei were stained using Hoechst 33258 (Sigma). For both readouts, images were acquired using 10x objective with nine imaged fields per well and analysed using the manufacturer's software (PerkinElmer, Columbus v.2.9.0.1546). For digital phase, the analysis sequence started with cell detection (method: c, common threshold: 0.05 , area $>100 \mu \mathrm{m}^{2}$, splitting coefficient: 6.5 , individual threshold: 0.05 , contrast $>0.05$ ) and was followed by calculating morphology, intensity and position properties as well as cell confluence. Our analysis showed good concordance between digital phase and nuclei staining based endpoints. Well level data were analysed using ActivityBase (IDBS) and R (v.3.6.1). Test well results were normalized relative to the corresponding intra-plate control (no virus assigned as $100 \%$ inhibition of cellular toxicity, with virus assigned as $0 \%$ inhibition of cellular toxicity). Outliers were eliminated according to 3-sigma method. Plate level statistical performance was assessed using the standard Z' calculation ${ }^{5}$. Dose response curves fitted in GraphPad Prism v.8.0.0 (GraphPad Software) using a 4-parameter logarithmic (least squares fit).

\section{Compound toxicity against Caco- 2 cells at 48 hours}

Cellular toxicity was detected using intracellular ATP concentrations. Day -1: Caco-2 cells were harvested using Acutase followed by re-suspension to the required number of 2000 cells/well in cell culture media (EMEM $+10 \%$ FCS, $1 \%$ non-essential amino acids $2 \mathrm{mM} \mathrm{L}$-glutamine, $100 \mathrm{U} / \mathrm{ml}$ penicillin and $0.1 \mathrm{mg} / \mathrm{ml}$ 
streptomycin, $1 \mathrm{mM}$ sodium pyruvate). $20 \mu \mathrm{L}$ /well were transferred in white solid 384 well CellStar plates (GreinerBio) and incubated for $20 \mathrm{~h}$ at $37^{\circ} \mathrm{C}, 5 \% \mathrm{CO}$. Day 0: Compounds were added to cells using Echo $550 \mathrm{R}$ Liquid Handler. Assay plates were incubated for additional $48 \mathrm{~h}$ at $37^{\circ} \mathrm{C}, 5 \% \mathrm{CO} 2$. One assay plate (day 0 plate) was measured using CellTiter-Glo Luminescent Cell Viability Assay (Promega Inc.), this plate is used for baseline measurement of cell viability. Day 2: CellTiter-Glo Luminescent Cell Viability Assay reagents were prepared according to manufacturer protocol. Assay plates were equilibrated for $10 \mathrm{~min}$ at room temperature. The reaction is initiated by addition of $10 \mu \mathrm{L} /$ well of the CellTiter-Glo Reagent to cells. Assay plates were centrifuged for $1 \mathrm{~min}$ at $700 \mathrm{xg}$ and incubated for $10 \mathrm{~min}$ in the dark at RT prior to measurement. Luminescence was detected on the EnVision plate Reader (0.05 s measurement time) (Perkin Elmer). Data analysis was performed using ActivityBase (IDBS), outliers were eliminated according to 3-sigma method. Compound data is normalised to the high (included $0.5 \% \mathrm{v} / \mathrm{v}$ DMSO - 100 $\%$ viability) and low control ( $25 \mu \mathrm{M}$ Sodium Selenite - $0 \%$ viability $)^{6}$.

\section{Protocols for KNIME v3.7.1 in the data analysis workflows2 cells at 48 hours}

KNIME v.3.6.1 (KNIME) ${ }^{7}$ has been used to compare external compound sets and screened compounds. Structural identity/similarity were used to select compounds. MACCS fingerprints generated within KNIME with RDKit were used for comparison and the Tanimoto index to measure similarity.

\section{Availability of data sets}

Workflow and R Scripts will be made available in https://github.com/agiani99/KNIME_Screen Primary and dose response data will be made available via ChEMBL (https://www.ebi.ac.uk/chembl/) Images will be made available via the image data archive (IDR) (https://idr.openmicroscopy.org/)

\section{Table}

Please see the supplementary files section to view the table.

\section{Figures}




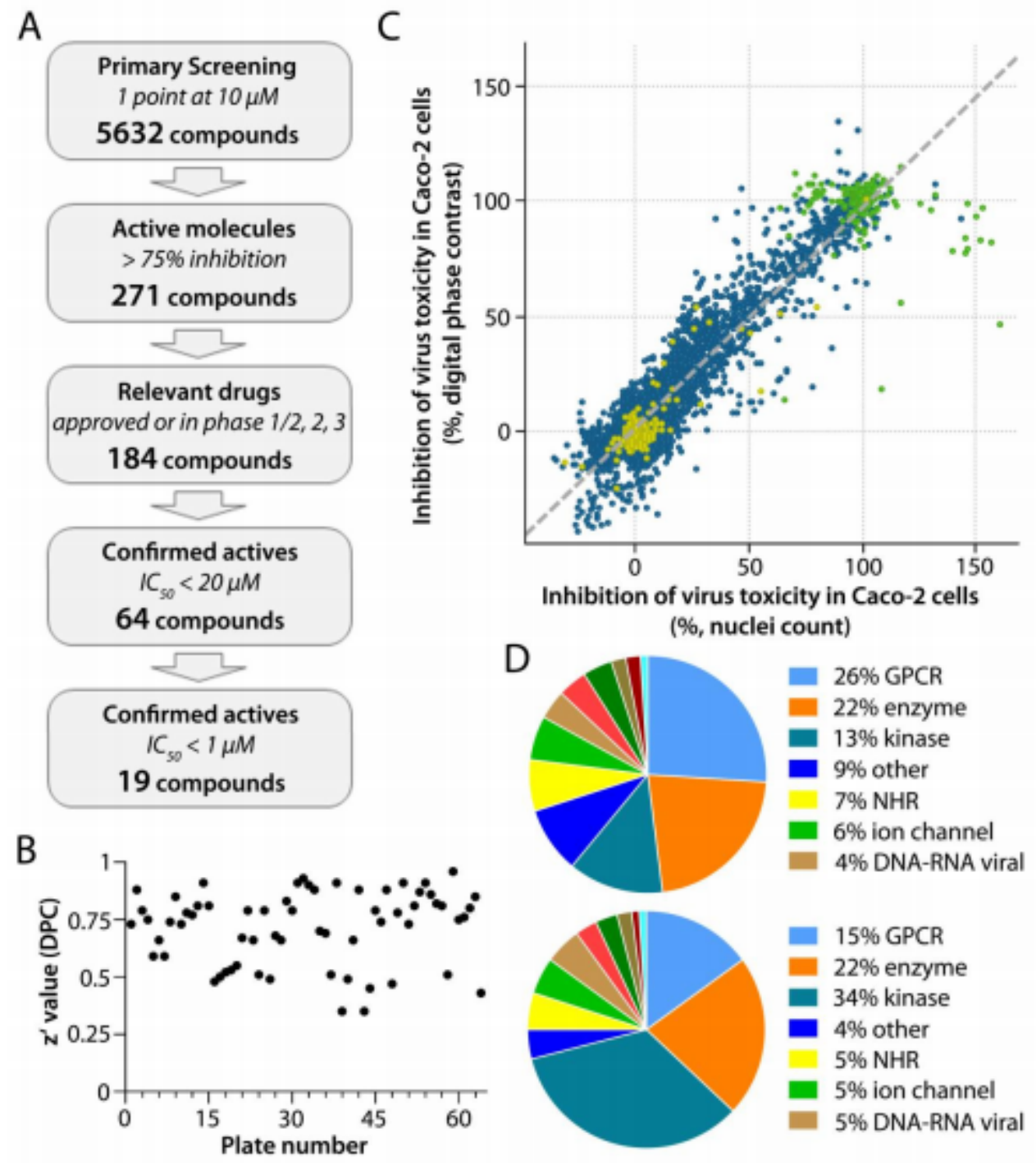

\section{Figure 1}

Primary screening results. A) Primary screen statistics and numbers of analysed compounds. B) Assay quality statistic, Z,' versus screening order in the Primary screens. C) Virus inhibition (\%) based on cell number for fluorescent nuclei readout (x-axis) and digital phase readout ( $y$-axis digital phase) Key: Blue (compound); yellow (100\% inhibition in the presence of virus), Green ( $0 \%$ inhibition in the absence of virus). D) Primary therapeutic target classes for all screened compounds (upper panel) compared to the hit population above $75 \%$ cut-off (lower panel).

\section{Supplementary Files}

This is a list of supplementary files associated with this preprint. Click to download.

- 2521570supp4542676q8hnwd.pdf

- Table1.docx 